\title{
Guiding and Focusing of Electromagnetic Fields with Wedge Plasmon Polaritons
}

\author{
Esteban Moreno, ${ }^{1 *}$ Sergio G. Rodrigo, ${ }^{2}$ Sergey I. Bozhevolnyi, ${ }^{3}$ L. Martín-Moreno, ${ }^{2}$ and F. J. García-Vidal ${ }^{1}$ \\ ${ }^{1}$ Departamento de Física Teórica de la Materia Condensada, Universidad Autónoma de Madrid, E-28049 Madrid, Spain \\ ${ }^{2}$ Departamento de Física de la Materia Condensada, Universidad de Zaragoza-CSIC, E-50009 Zaragoza, Spain \\ ${ }^{3}$ Department of Physics and Nanotechnology, Aalborg University, DK-9220 Aalborg Ost, Denmark \\ (Received 4 June 2007; revised manuscript received 20 November 2007; published 14 January 2008)
}

We study theoretically electromagnetic modes guided by metallic wedges at telecom wavelengths. These modes are found to exhibit superior confinement while showing similar propagation loss as compared to other subwavelength guiding configurations. It is also shown that mode focusing can be realized by gradual modification of the wedge geometry along the mode propagation direction.

DOI: 10.1103/PhysRevLett.100.023901

PACS numbers: 42.79.Gn, 42.25.Bs, 73.20.Mf

Downscaling optical circuits remains a major issue in micro and nanotechnology. The realization of subwavelength guiding structures is a key factor for miniaturization, because these components would permit denser waveguide packaging without crosstalk, and lower waveguide bending loss. Surface plasmon polaritons (SPPs) sustained by metallic structures are one of the most promising approaches to achieve modal sizes smaller than the wavelength. Various geometries have been proposed to fulfill this goal [1-3]. The main design difficulty is finding structures that support electromagnetic fields with small modal size while keeping a high propagation length. Channel plasmon polaritons (CPPs) [4] supported by $V$-shaped metallic grooves fulfill these requisites to a certain extent and devices based upon them have been experimentally demonstrated [5] at telecommunication wavelengths. CPP modes present relatively low losses when operating very close to modal cutoff, but their modal size is in this case larger than expected [6].

In this Letter we study the modes supported by a metallic wedge [termed wedge plasmon polaritons (WPPs)]. It is found that WPPs, while showing significantly smaller modal size than CPPs, exhibit similar propagation length as CPPs. We also suggest how a plane SPP propagating along a flat surface can be coupled to a tightly confined WPP mode by using a gradual deformation of the surface profile.

The basic structure studied here is a metallic wedge surrounded by vacuum. It has an infinitely long edge, which is the propagation direction ( $Z$ axis) for the supported electromagnetic modes (the edge is rounded with radius of curvature $r$ ). The wedge angle is denoted as $\phi$. We consider both wedges truncated at a certain height $y=$ $h$ [as shown in the inset of Fig. 1(b)], and nontruncated wedges $(h \rightarrow \infty)$. The modes corresponding to nontruncated wedges will be named $\operatorname{WPP}(\infty)$. The considered metal is gold, which is represented by a Drude-Lorentz type dielectric function [7]. The size of the considered structures is sufficiently large so as to use bulk dielectric functions and neglect additional damping due to electron scattering at the metal surface. The effective index model allows one to argue that a metallic wedge sustains modes that are localized close to its edge and propagate along it, but numerical simulations are needed to determine accurately the modal characteristics. The results presented in
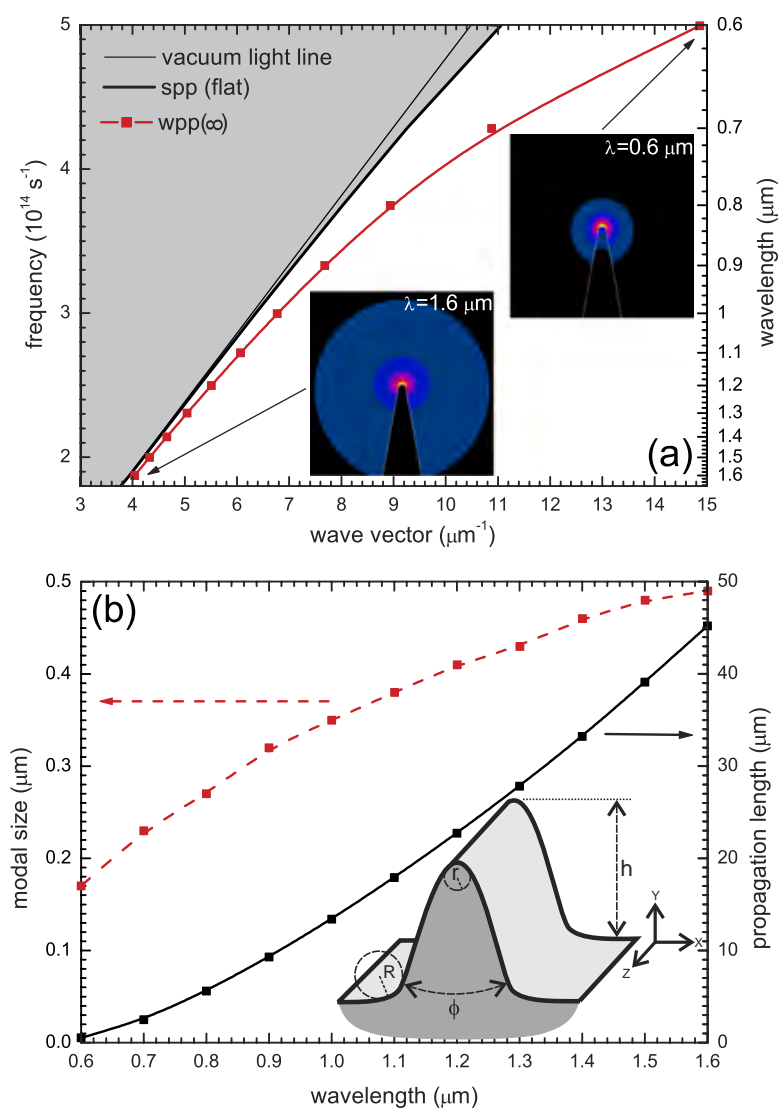

FIG. 1 (color online). (a) WPP dispersion relation. Black thick line, SPP mode on a flat surface; red or gray line (squares), $\mathrm{WPP}(\infty)$ mode supported by a nontruncated wedge. Insets: timeaveraged electric field of $\operatorname{WPP}(\infty)$ mode at wavelengths $\lambda=$ $0.6 \mu \mathrm{m}$ and $\lambda=1.6 \mu \mathrm{m}$. The lateral size of the insets is $0.5 \mu \mathrm{m}$. (b) Modal size (red or gray dashed line) and propagation length (black solid line) of WPP $(\infty)$ mode as a function of $\lambda$. Inset: diagram of the truncated wedge. 
this Letter have been obtained with the multiple multipole (MMP) method [8] and the finite difference time domain (FDTD) technique [9]. We used MMP for the structures with cylindrical symmetry and FDTD for the structures with a more complex geometry. Very good agreement between the results of both methods is obtained.

After an early analysis of WPPs in the electrostatic approximation [10], these modes were recently studied by Pile et al. [11] in the visible regime, where the mode propagation length is very short. In our Letter the emphasis is on telecom wavelengths where losses are much lower (see also [14]). Figure 1 displays the modal behavior of $\operatorname{WPP}(\infty)$ modes for a wedge with angle $\phi=20^{\circ}$ and radius of curvature of the edge $r=10 \mathrm{~nm}$. Panel (a) shows the dispersion relation (red or gray line) of the fundamental mode. As corresponds to a nonradiative mode, it lies outside the shaded area bounded by the dispersion relation of a SPP mode. The mode has no cutoff wavelength. The modal shape for wavelengths at both ends of the considered spectrum is plotted in the insets. Modal size and propagation length $\left(l=\left[2 \operatorname{Im}\left(k_{z}\right)\right]^{-1}, k_{z}\right.$ being the modal wave vector) as a function of wavelength are presented in panel (b) (left and right axes, respectively). Here the modal size is defined as the transverse separation between the locations where the electric field amplitude of the mode has fallen to one tenth of its maximum value. The factor $1 / 10$ in this definition is somehow arbitrary but it is sufficient for our mode characterization purposes. The modal size (red or gray dashed line) grows as $\lambda$ increases, but subwavelength guiding is achieved in the whole regime. As mentioned above, the propagation length (black line) is very short in the visible region of the spectrum $(\simeq$ $0.5 \mu \mathrm{m}$ at $\lambda=0.6 \mu \mathrm{m}$ ), but it rises to about $40 \mu \mathrm{m}$ in the telecom regime.

Wedge and channel plasmon polaritons are now compared at $\lambda=1.5 \mu \mathrm{m}$. In order to have a meaningful comparison, we consider exactly the same geometry for both structures, simply exchanging the metallic and vacuum regions (Fig. 2). The angle and radius of curvature are the same as above, but we now compute more realistic
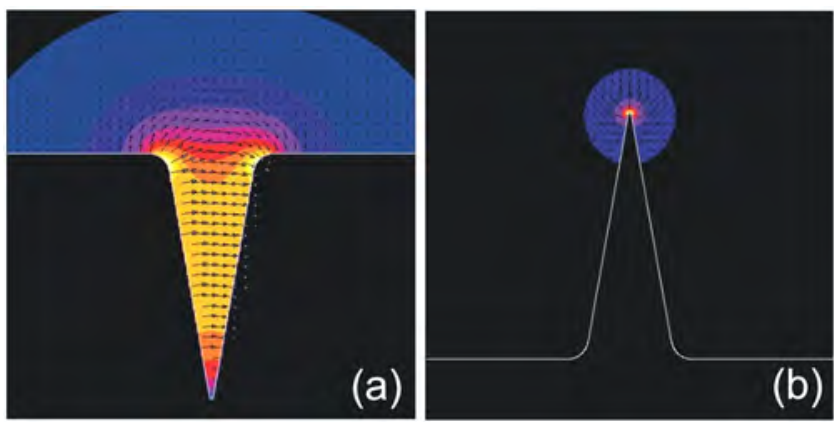

FIG. 2 (color online). Transverse electric field of (a) CPP mode, and (b) WPP mode, both at $\lambda=1.5 \mu \mathrm{m}$. The geometry of both structures is identical (see main text) exchanging the metallic and vacuum regions. The panel's lateral size is $2 \mu \mathrm{m}$. structures with finite height $(h=1.2 \mu \mathrm{m}$, a typical value for experiments with CPPs). The corners where the flat horizontal surface meets the triangular structure are also rounded (with a radius of curvature $R=100 \mathrm{~nm}$, also typical for experimental CPPs). The transverse electric field of both CPP and WPP modes is plotted in Fig. 2. The modal size of the WPP is $0.46 \mu \mathrm{m}$, significantly smaller than that of the CPP $(2.5 \mu \mathrm{m})$. This is mainly due to the fact that the CPP mode is hybridized with wedge modes supported by the edges at both sides of the groove [6]. These edges correspond to wedges with a large angle $\left(\phi^{\prime}=100^{\circ}\right)$ and radius of curvature $(R=100 \mathrm{~nm})$, and for which the corresponding WPP modal sizes are larger, as will be shown below. Despite the different modal sizes, the computed propagation lengths are quite similar for both modes: $37 \mu \mathrm{m}$ for the WPP, and $34 \mu \mathrm{m}$ for the CPP. It is worth mentioning that the CPP mode is very close to cutoff and for a height $h=1 \mu \mathrm{m}$ the mode is no longer guided, whereas the WPP is guided whenever the height verifies $h>h_{c} \simeq 0.2 \mu \mathrm{m}$.

Let us now consider the dependence of the modal characteristics of WPPs as a function of the most relevant geometric parameters. The following data correspond to $\lambda=1.5 \mu \mathrm{m}$. The dependence with the height $h$ of the wedge is summarized in panels (a) and (b) of Fig. 3, whereas the dependence with the angle $\phi$ of a $\operatorname{WPP}(\infty)$ is presented in the lower panels (c) and (d). The modal
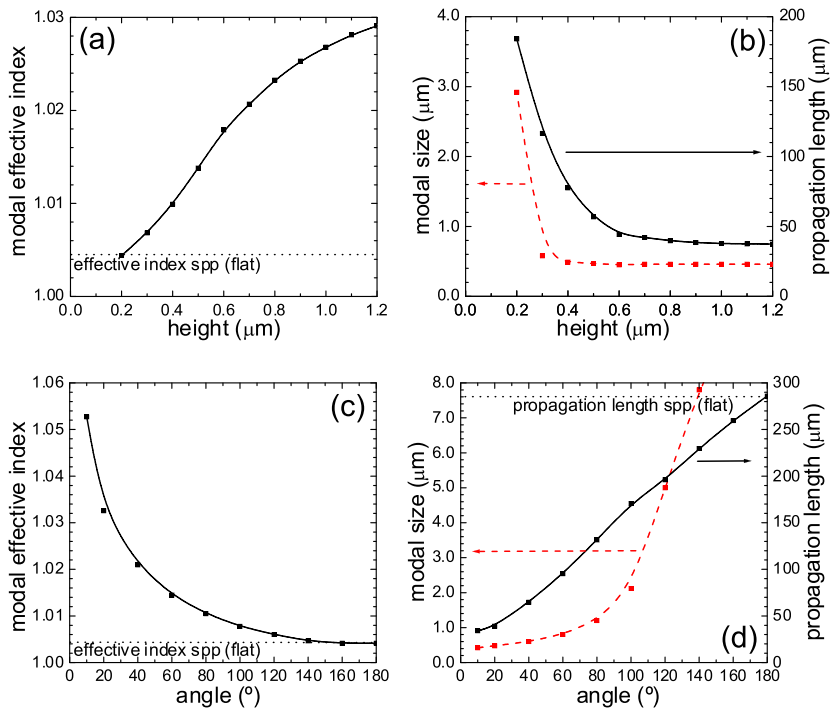

FIG. 3 (color online). WPP modal characteristics as a function of wedge height or angle. In (a) and (b) the height is varied and the wedge angle is constant, $\phi=20^{\circ}$. In (c) and (d) the wedge angle is varied (the wedge is not truncated). The radius of curvature is $r=10 \mathrm{~nm}$ in all cases. (a) Solid line, effective index of WPP mode. (b) Red or gray dashed line, modal size of WPP; solid line, propagation length of WPP. (c) Solid line, effective index of WPP $(\infty)$ mode. (d) Red or gray dashed line, modal size of $\operatorname{WPP}(\infty)$; solid line, propagation length of $\mathrm{WPP}(\infty)$. 
effective index $n_{\text {eff }}$ (i.e., modal wave vector divided by wave vector in vacuum) is displayed in panel (a). As $h$ decreases, $n_{\text {eff }}$ tends to the effective index of a SPP on flat surface (for $h<h_{c}, n_{\text {eff }}$ reaches the effective index of a SPP and the mode is no longer guided). Note that a low effective index is equivalent to a more extended field, as confirmed in panel (b) where the modal size is plotted (red or gray dashed line). The propagation length is also shown in panel (b) (black line), increasing when the cutoff height is approached. The behavior of the $\operatorname{WPP}(\infty)$ modal characteristics as the angle $\phi$ increases is reminiscent to what occurs when the height $h$ decreases. There is, however, a major difference: there is no critical angle above which the mode is no longer guided. As $\phi$ is increased towards $180^{\circ}$, propagation length, $n_{\text {eff }}$, and modal size tend to those of a SPP on a flat surface. Modal size rapidly increases as the angle grows, but our numerical simulations show waveguiding no matter how large the angle is (whenever $\phi<$ $180^{\circ}$ ).

Up to this point we have been concerned only with waveguiding, finding that WPPs display good confinement and propagation length. In the rest of the paper we present strategies for WPP $\leftrightarrow$ SPP conversion. A device with this functionality should convert the shape and size of a WPP to that of a SPP. The task can be also understood as focusing an SPP to a WPP (if the time arrow is reversed). Since SPPs on flat surfaces have infinite transverse extension, the main challenge is the large modal mismatch. Here, conversion shall be achieved by deforming the metal surface from a wedge geometry to a flat geometry in a continuous way along the mode propagation direction ( $Z$ axis). In other words, the wedge height or angle become functions of the $z$ coordinate, $h(z), \phi(z)$. It is clear from Figs. 3(a) and 3(c) that, as the height $h$ shrinks or the angle $\phi$ increases, the effective index is reduced, leading to a growth of the modal size. If this conversion is performed slowly, radiation and reflection during the process should be low. In order to verify this scenario we have performed three-dimensional (3D) FDTD simulations for structures with constant wedge angle $\left(\phi=20^{\circ}\right)$ and various $h(z)$ profiles. The tight confinement of the mode requires very fine meshes (we used a mesh of $10 \mathrm{~nm}$ ). The simulation domain is a parallelepiped surrounded by perfect matched layers (PMLs). The simulations are performed at $\lambda=1.5 \mu \mathrm{m}$ in continuous-wave mode. All structures have an initial section with constant wedge height. The input WPP mode is excited by a source (located at $z=0.5 \mu \mathrm{m}$ in front of the wedge and buried inside a cavity to prevent direct illumination from the source to the conversion device). After a short transient $\left(z<z_{t}=2 \mu \mathrm{m}\right)$, the field settles down to a propagating WPP mode. We first simulate a set of test structures (structures I) all of them with constant height, $h \in$ $(0.2,0.48) \mu \mathrm{m}$. These computations will allow evaluation of losses in later structures, and served as a test of the FDTD simulations (comparison with MMP results is very good). After the initial transient, the found exponential modal decay is solely due to absorption in the metal (and not in the PMLs). Modal reflection at the domain boundary is very small (reflected power less than $0.1 \%$ ). Thus, for structures I, the field computed at $z=9.0 \mu \mathrm{m}$ (shortly before the simulation domain boundary) is a pure WPP mode without radiation. Structure II has $h=0.48 \mu \mathrm{m}$ for $z<3.9 \mu \mathrm{m}$, and then the wedge height decreases linearly to zero along a distance $\Delta z=3.3 \mu \mathrm{m}[h(z)$ is zero beyond this point]. Figures 4(a) and 4(b) show longitudinal cross sections $[Z Y$ plane (side view), and $Z X$ plane at a height $h=0.3 \mu \mathrm{m}$ (top view), respectively] of the electric field amplitude. The mode runs from left to right. From panels (a) and (b) reflection seems to be very low. As the wedge height decreases, the modal size expands. This is observed in panels (c) to (f), which display the corresponding transverse cross sections ( $X Y$ planes at various $z$ coordinates). For comparison, we have considered structure III, which is identical to structure II for $z<$ $4.6 \mu \mathrm{m}$ but with a wedge height abruptly becoming zero after this point [side and top view in panels $(\mathrm{g})$ and $(\mathrm{h})$, respectively]. In this case we observe strong reflection at the discontinuity. We now want to evaluate non-Ohmic losses during conversion in structure II. To this end, for
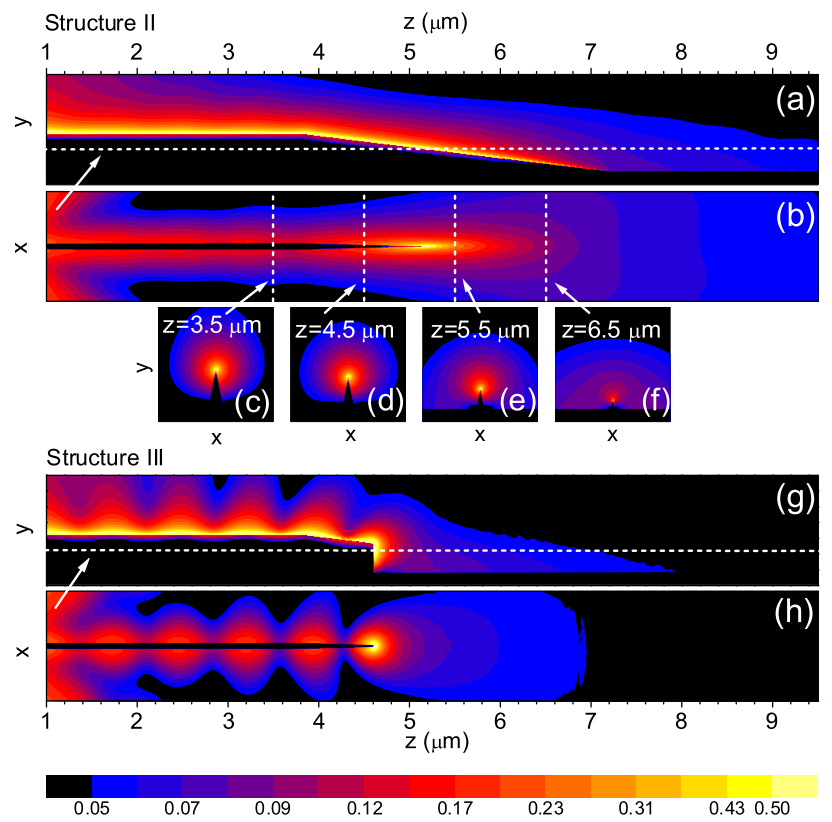

FIG. 4 (color online). Electric field amplitude in the WPP $\rightarrow$ SPP transition (geometries are detailed in the main text). (a) and (b) longitudinal cross sections for structure II [dashed line in (a) is the location of longitudinal section (b)]. (c), (d), (e), and (f) transverse cross sections ( $X Y$ plane, at various $z$ coordinates) for structure II [dashed lines in (b) show the position of these transverse cross sections]. (g) and (h) longitudinal cross sections for structure III. The Cartesian axes are shown in Fig. 1(b). The wavelength is $\lambda=1.5 \mu \mathrm{m}$. The size of all panels along $X$ and $Y$ directions is $1.4 \mu \mathrm{m}$. 

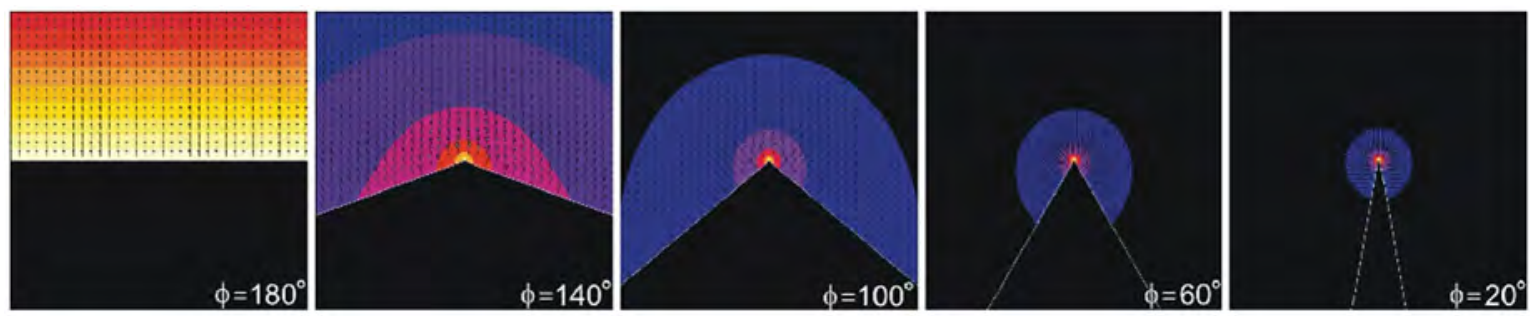

FIG. 5 (color online). Focusing of the WPP $(\infty)$ modal field as the wedge angle $\phi$ is slowly decreased from $\phi=180^{\circ}(\mathrm{SPP}$ mode on a flat surface) to $\phi=20^{\circ}$. The panels display the transverse electric field at a wavelength of $1.5 \mu \mathrm{m}$. The lateral size of the panels is $2 \mu \mathrm{m}$. The radius of curvature of the tip is $r=10 \mathrm{~nm}$. The color scale or shading of the various panels is not the same.

every transverse cross section $z<z_{c} \simeq 5.8 \mu \mathrm{m}\left(z_{c}\right.$ being the coordinate corresponding to the modal cutoff height $h_{c}$ ), we have computed the overlap [12] of the field in structure II at $z$, and the field of a WPP mode with the height of structure II at $z$ (these fields are available from our computations of structures I). Whenever WPP reflection occurs, the overlap integral as a function of $z$ displays ripples due to the formation of a standing wave. From this modulation we estimate that reflection is about $0.2 \%$ (a similar analysis for structure III gives a value of $20 \%$ ). Additionally, when power is radiated, the value of the overlap is reduced because, due to orthogonality, radiation modes are projected out when the overlap is computed. The results of this test (once absorption losses are taken into account) show that the power guided by the WPP is preserved for $z_{t}<z<z_{c}$ thus confirming that very little power is radiated after the transient (less than 5\%). We can thus conclude that structure II achieves its design purpose up to the coordinate $z=z_{c}$. For $z>z_{c}$ no WPP mode exists and the field extends outside the simulation window. However, quantitative comparison of the field computed for structure II in the transverse cross section at $z=9 \mu \mathrm{m}$ and that of a pure SPP shows that the field is mainly a SPP at this coordinate. Thus, the power through this cross section (which amounts to $29 \%$ of the input power) is essentially carried by SPPs. This is a lower bound estimation for the WPP $\rightarrow$ SPP conversion as the finite size of the simulation domain impedes the calculation of the total power coupled to SPPs propagating in all directions in the horizontal plane.

In structure II, WPP modes do not exist for $h<h_{c}$. Moreover, such a device converts a WPP mode to SPPs propagating with an in-plane angular spectrum. For both reasons the coupling of WPP to Z-propagating SPP may be reduced. These restrictions should not apply for $\operatorname{WPP}(\infty)$ modes when the angle is the control parameter along the $Z$ axis. Therefore, we expect a better performance for a structure where the wedge angle $\phi$ is continuously varied from a flat surface $\left(\phi=180^{\circ}\right)$ to a wedge with $\phi=20^{\circ}$. Three-dimensional simulations of this case are inherently very difficult because, by construction, the modal size grows increasingly fast as $\phi \rightarrow 180^{\circ}$. Thus, the mode cannot fit in the simulation domain, which is constrained by the available computer memory. Nevertheless, the idea is illustrated in Fig. 5, that plots the transverse electric field for decreasing wedge angles (2D MMP simulations). Note how the field is concentrated close to the edge. The previously shown FDTD computations and other studies [13], lead us to expect that focusing (without radiation or reflection) can be achieved in a short length also in this case.

In conclusion, we have shown that WPPs are good subwavelength guiding structures. At telecom wavelengths, their guiding properties were found superior to the ones offered by CPPs. We have also explored the possibility of light focusing via the geometry-driven conversion of a standard SPP into a tightly confined WPP.

Funded by the EU (No. FP6-2002-IST-1-507879) and Spanish MEC (No. MAT2005-06608-C02).

Note added. - The work of Yan and Qiu [14] was published during the review process.

*esteban.moreno@uam.es

[1] J. Takahara et al., Opt. Lett. 22, 475 (1997).

[2] P. Berini, Opt. Lett. 24, 1011 (1999).

[3] D. K. Gramotnev and D. F. P. Pile, Appl. Phys. Lett. 85, 6323 (2004).

[4] I. V. Novikov and A. A. Maradudin, Phys. Rev. B 66, 035403 (2002).

[5] S. I. Bozhevolnyi et al., Phys. Rev. Lett. 95, 046802 (2005); S. I. Bozhevolnyi et al., Nature (London) 440, 508 (2006).

[6] E. Moreno et al., Opt. Lett. 31, 3447 (2006).

[7] A. Vial et al., Phys. Rev. B 71, 085416 (2005).

[8] C. Hafner, Post-Modern Electromagnetics (Wiley, Chichester, 1999).

[9] A. Taflove and S. Hagness, Computational Electrodynamics: The Finite-Difference Time-Domain Method (Artech House, Boston, 2000).

[10] L. Dobrzynski and A. A. Maradudin, Phys. Rev. B 6, 3810 (1972).

[11] D. F. P. Pile et al., Appl. Phys. Lett. 87, 061106 (2005).

[12] See EPAPS Document No. E-PRLTAO-100-024802 for the definition of overlap integrals. For more information on EPAPS, see http://www.aip.org/pubservs/epaps.html.

[13] S. A. Maier et al., Phys. Rev. Lett. 97, 176805 (2006).

[14] M. Yan and M. Qiu, J. Opt. Soc. Am. B 24, 2333 (2007). 